\title{
Vertical advection of phytoplankton and productivity estimates: a dimensional analysis
}

\author{
Charles L. Gallegos ${ }^{1} \&$ Trevor Platt $^{2}$ \\ ${ }^{1}$ Water Quality and Watershed Research Laboratory, P.O. Box 1430 Durant, Oklahoma 74701, USA \\ ${ }^{2}$ Marine Ecology Laboratory, Bedford Institute of Oceanography, Dartmouth, Nova Scotia B2Y 4A2, Canada
}

\begin{abstract}
Standard incubation procedures may underestimate phytoplankton productivity by maintaining the organisms at fixed light intensities or depths. Conditions under which bias could occur are quantified in terms of 2 dimensionless groups. The first is a dimensionless time, $t^{*}=k^{2} K_{z} t$, where $\mathrm{k}=$ diffuse attenuation coefficient for light, $\mathrm{K}_{\mathrm{z}}=$ coefficient of vertical eddy diffusivity, $\mathrm{t}=$ incubation time. It represents the duration of the incubation scaled to the time required for a freely-circulating ensemble of phytoplankton to diffuse 1 optical attenuation length. The second is a photoinhibition index, $\left(\mathrm{I}_{\mathrm{b}}\right)^{-1}=\mathrm{I}_{0} / \mathrm{I}_{\mathrm{b}}$, where $\mathrm{I}_{0}=$ surface incident irradiance, $\mathrm{I}_{\mathrm{b}}$ is a parameter characterizing the photoinhibition region of the light saturation curve of photosynthesis; photoinhibition at surface intensities is 0 when $\left(I_{b}{ }^{*}\right)^{-1}=0$, and increases as $\left(I_{b}{ }^{*}\right)^{-1}$ increases. Published data on the effects of vertical motion on phytoplankton photosynthesis indicate that $\left(\mathrm{I}_{b}{ }^{*}\right)^{-1}$ must exceed a critical value for negative bias to occur in productivity estimates. In general, the critical value is a function of $t^{\circ}$, but certain boundaries can be identified. A minimum critical value of $\left(\mathrm{I}_{\mathrm{b}}{ }^{\circ}\right)^{-1}=0.25$ (i.e. low susceptibility to photoinhibition), below which there is no bias, occurs at $t^{\circ} \simeq 10$. For $\left(\mathrm{I}_{6}{ }^{\circ}\right)^{-1}>1.5$ (high susceptibility to photoinhibition), some bias occurs for all values of $t^{*}$ Values of $\left(I_{b}{ }^{*}\right)^{-1}$ are examined in 4 regions to infer the degree to which previous data sets may have been biased.
\end{abstract}

\section{INTRODUCTION}

It is well known that the environment of phytoplankton fluctuates on a wide range of temporal and spatial scales. This understanding has led to the realization that standard techniques for measuring rate processes in aquatic environments do not necessarily resolve events on the spatial and temporal scales perceived by the organisms. An example is the standard in situ light and dark bottle method for measuring primary productivity in marine and freshwater habitats. It has been suggested that this method has led to serious underestimates of photosynthetic rates because the phytoplankton are maintained at fixed light intensities (Harris 1978,1980 ). It is argued that this practice not only exaggerates surface inhibition, but also that the method measures an average, steady-state rate at saturating intensities that is not necessarily representative of the rate achieved by cells experiencing the full range of turbulent motions in their natural setting.

Such arguments are based on ecological reasoning, and on laboratory and field experiments. Realization that the light field of phytoplankton fluctuates on a number of time scales suggests the possibility that high frequency light fluctuations, much shorter than the typical incubation time, may be a feature of the environment to which certain species have a positive adaptation. Experiments using concentrated field populations (Harris \& Piccinin 1977) and laboratory phytoplankton cultures (Marra 1978a, 1980), show elevated rates of photosynthesis in the first few minutes after initial exposure to saturating or inhibiting irradiance, and so support this view.

Although a number of workers have attempted to simulate vertical motion in incubation experiments, the effects on the primary productivity estimates have been equivocal. Jewson \& Wood (1975) pumped lakewater up and down through glass tubing and found both elevated and depressed rates of integrated photosynthesis relative to estimates of photosynthesis in stationary bottles. Marra (1978b) simulated motion within Langmuir cells and found higher rates of production in the circulated bottles in 4 of 6 cases. Gallegos \& Platt (1982) simulated the effects of both organized motion in Langmuir cells and diffusive transport in a light gradient, where the degree of dispersion could be scaled to the momentum flux, if it were known. Results from the eastern Canadian Arctic 
indicated that vertical motion affected productivity estimates only when shade-adapted cells were entrained into the surface layer during mixed-layer deepening.

In this paper we attempt to account for some of the variability in results of such experiments. We first quantify the conditions that must exist in the environment, the organisms, and the method to produce biased estimates of productivity when the temporal variability of the light field is ignored. Two conditions necessary to produce discrepancies in production rates between mixed and statically contained populations can be readily identified. First, the light field to which the freely circulating population is exposed must be substantially more variable than that experienced by the contained population. Second, the photosynthetic performance of phytoplankton in fluctuating light must be different from the response measured in constant light. In the following sections we derive indices to quantify these conditions, and emphasize the kinds of measurements that may be made to evaluate the physical conditions at sampling time. The effect of fluctuating light on photosynthesis is formulated in terms of a photoinhibition index. We then examine existing data on photosynthesis in fluctuating light to determine critical values of variance in the light field and susceptibility to photoinhibition needed to produce biased estimates of productivity. Using the identified criteria data, sets from several regions are examined to determine the frequency with which the photoinhibition criterion has been exceeded; violation of the photoinhibition criterion alone (without knowledge of the physical conditions during the incubation) is a necessary but not sufficient condition for biased productivity estimates.

\section{DIMENSIONAL ANALYSIS}

The light field of phytoplankton. An individual phytoplankter receives light in a Lagrangian framework: fluctuations in light at the cell occur as a result of both changes in surface-incident irradiance and of motion in the vertical light gradient. Phytoplankton incubated in situ or in simulated in situ boxes are exposed to the fluctuations in surface incident irradiance, appropriately attenuated. For these techniques, the deviation of the light field experienced by the contained population from that of the freely circulating population depends on the magnitude of the changes due to advection, which are eliminated by the incubation procedure, relative to changes in surface light, which are incorporated into the incubation. The light, $I_{z}$ impinging on a cell at depth $z$ is approximated as

$$
\mathrm{I}_{2}=\mathrm{I}_{0} \exp (-\mathrm{kz})
$$

where $\mathrm{I}_{0}=$ surface-incident irradiance; $\mathrm{k}=$ the diffuse attenuation coefficient. On the time scale of typical productivity incubations ( 2 to $24 \mathrm{~h}$ ), $\mathrm{k}$ may be considered constant while $I_{0}$ and a cell's position, $z$, vary. (Although not strictly true for incubations of 12 to $24 \mathrm{~h}$, changes in $k$ on these time scales should be small compared either to changes in $\mathrm{I}_{0}$ or to changes in a cell's position.) Log-transformation of (1) separates light fluctuations arising from changes in $\mathrm{I}_{0}$ (i.e. Eulerian) from those arising by changes in a cell's position (i.e. advective),

$$
\ln \left(I_{z}\right)=\ln \left(I_{0}\right)-k z
$$

The total variance in $\ln \left(\mathrm{I}_{z}\right)$ is then

$\operatorname{Var}\left[\ln \left(I_{z}\right)\right]=\operatorname{Var}\left[\ln \left(I_{0}\right)\right]+k^{2} \operatorname{Var}[z]-2 k \operatorname{Cov}\left(I_{0}, z\right)$

where Var[z] represents the variance of a phytoplankter's vertical position and this variance is not the same as the variance of the depth distribution of phytoplankton biomass. The covariance term on the right hand side of Eq. (3) could be significant in the case of positive or negative phototaxis of a phytoplankter. For simplicity, we must restrict attention to well-mixed surface layers in which a cell's sinking or swimming speed is negligible compared to turbulent transport. Further, $\operatorname{Var}[\mathrm{z}]$ and $\ln \left(\mathrm{I}_{0}\right)$ could be coupled through the effects of solar heating on the quenching of turbulence, but inclusion of such effects would require a more elaborate mixed-layer model (see e.g. Simpson \& Dickey 1981). For our purposes, if $\operatorname{Var}\left[\ln \left(\mathrm{I}_{0}\right)\right]>\mathrm{k}^{2} \operatorname{Var}[z]$, then most of the variability in light at the cell surface is due to variations in incident light, and in situ or simulated in situ incubations adequately represent the temporal variability of the light field; conversely, if, on the time scale of a productivity incubation, $\operatorname{Var}\left[\ln \left(\mathrm{I}_{0}\right)\right]<<$ $k^{2} \operatorname{Var}[z]$, serious alteration of the light field may arise from static containment of the phytoplankton.

Rough estimates of the Eulerian and advective components of the variance in light may be made using some simplifying assumptions. Many investigators employ short ( 2 to $6 \mathrm{~h}$ ) incubations centered about solar noon to minimize 1 kind of error. Under such conditions, the major source of variation in $\ln \left(\mathrm{I}_{0}\right)$ is the passage of scattered clouds. Assume that var[ $\left[\ln \left(\mathrm{I}_{0}\right)\right]$ is governed largely by the extremes of irradiance from when the sun is unobstructed to when it is completely obscured by a cloud, and that the irradiance reaching the water surface when the sun is obscured is a fraction, $a$, of the unobstructed irradiance, $\mathrm{I}_{0}{ }^{\prime}$. Let $\mathrm{f}=$ fraction of cloud cover (a standard meteorological observation $)_{;}$then $\ln \left(\mathrm{I}_{0}\right)$ is a random variable that takes on the value $\ln \left(I_{0}{ }^{\prime}\right)$ with probability $(1-f)$, and the value $\ln (a)$ $+\ln \left(\mathrm{I}_{0}{ }^{\prime}\right)$ with probability $\mathrm{f}$. The mean of $\ln \left(\mathrm{I}_{0}\right)$ is

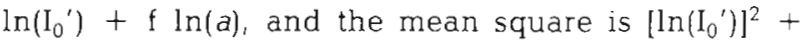


$2 f \ln (a) \ln \left(I_{0}\right)+f[\ln (a)]^{2}$. From the definition of variance it follows that

$$
\operatorname{Var}\left[\ln \left(\mathrm{I}_{0}\right)\right]=\mathrm{f}(1-\mathrm{f})[\ln (a)]^{2}
$$

For a given value of $a, \operatorname{Var}\left[\ln \left(\mathrm{I}_{z}\right)\right]$ decreases quadratically from a maximum at $f=0.5$. From the data in Gallegos et al. (1977) and in Marra \& Heinemann (1982), a typical value for a would lie in the range 0.2 to 0.3 . Thus, the maximum contribution from scattered clouds to the specific variance in $\mathrm{I}_{0}$ should be about 0.65 . For incubations from sunrise to sunset, we assume that the variation of $\mathrm{I}_{0}$ is governed by the path of the sun, in which case $\mathrm{I}_{0}(\mathrm{t})$ may be approximated by a halfsine wave. The variance of $\ln \left(I_{0}\right)$ cannot be computed over the whole day because $I_{0}=0$ at sunrise and sunset. It is therefore necessary to compute the variance over some interval $(\delta, D-\delta)$, where $D=$ daylength. There is some leeway in the choice of $\delta$, but evidence indicates that when light fluctuations are confined to intensities below $I_{k}$ (the onset of light saturation), the time-integral of photosynthesis is adequately predicted by the relation derived from incubations in constant light (Harris \& Piccinin 1977, but cf. Frechette \& Legendre 1978). The time at which $\mathrm{I}_{0}$ first exceeds $\mathrm{I}_{\mathrm{k}}$ would be a conservative choice for $\delta$. Using a typical value for $\mathrm{I}_{\mathrm{k}}$ of $20 \mathrm{~W} \mathrm{~m}^{-2}$ (Platt \& Jassby 1976) and a maximum noon flux of $400 \mathrm{~W} \mathrm{~m}^{-2}, \delta$ would be such that $\operatorname{Var}\left[\ln \left(\mathrm{I}_{0}\right)\right]$ would be evaluated over $97 \%$ of the daylight period. In this example we find that $\operatorname{Var}\left[\ln \left(\mathrm{I}_{0}\right)\right]=0.46$, somewhat less than the maximum specific variance due to scattered clouds during short term incubations.

The advective contribution, $k^{2} \operatorname{Var}[z]$, to the specific variance in the light field during an incubation depends on both the diffuse attenuation coefficient and the intensity of vertical mixing; $k^{2} \operatorname{Var}[z]$ can therefore be expected to vary between regions and temporally within a region. The variance of the depth distribution of a phytoplankter depends on the duration of the incubation, $t$, by the relation

$$
\operatorname{Var}[z]=2 K_{z} t
$$

where $K_{z}=$ the coefficient of vertical eddy diffusivity. Eq. (5) assumes that Eulerian and Lagrangian statistics are equivalent (Taylor's hypothesis), a valid enough assumption for gross scale analysis of motion in a mixed layer (Denman \& Gargett 1983). Assuming 'typical' conditions for surface layers in temperate oceans, $\mathrm{k}=0.15 \mathrm{~m}^{-1}(30 \mathrm{~m}$ euphotic zone, Holmes 1957) and $\mathrm{K}_{\mathrm{z}}=10 \mathrm{~cm}^{2} \mathrm{~s}^{-1}$ (Oakey \& Elliott 1977), the advective contribution to the specific variance in the light field would be 0.68 for an incubation lasting $4 \mathrm{~h}$. This is roughly equivalent to the maximum expected contribution due to scattered cloudiness, and is somewhat greater than the contribution due to the diurnal variability over the period when $I_{0}(t)>I_{k}$. Other examples are shown in Table 1 . The expected variability in $2 \mathrm{k}^{2} \mathrm{~K}_{\mathrm{z}} \mathrm{t}$ is about 2 orders of magnitude within most habitats, and more than 4 orders of magnitude over all regions.

Eq. (5) suggests an alternative interpretation of the term $\mathbf{k}^{2} \operatorname{Var}[\mathbf{z}]$. Consider an ensemble of tagged phytoplankton released at some depth in a mixed layer. The time scale for the patch to diffuse over a distance of 1 optical attenuation length is given by $0.5 \mathrm{k}^{-2} \mathrm{~K}_{\mathrm{z}}^{-1}$. Thus, the product $2 \mathrm{k}^{2} \mathrm{~K}_{\mathrm{z}} \mathrm{t}$ (dimensionless) represents a time period (say the duration of the incubation) scaled to the time required for an ensemble of phytoplankton to spread vertically over a distance equal to 1 optical attenuation length. We use the symbol $\mathrm{t}^{*}$ to denote this dimensionless time.

To estimate at sampling time the degree of light variability due to advection requires knowledge of the particular flow situation. For wind-driven mixing in an unstratified layer, the coefficient of eddy diffusivity can be expressed as the product of a characteristic turbulent velocity, $w_{t}$ and a mixing length scale, $h$ (Denman \& Gargett 1983). Making this substitution, the second term on the right hand side of Eq. (3) can be now written as the product of 2 dimensionless groups: $k w_{t} t$ is the number of optical attenuation lengths through which a population would be advected by the characteristic velocity during the incubation, and $\mathrm{kh}$ the optical depth of the mixing layer. The relevant velocity scale is the shear velocity, $\mathrm{w}^{*}=\left(\tau_{0} / \mathrm{Q}\right)^{1 / 2}$, where $\tau_{0}$ is the surface shear stress and $Q$ is the density of water. The surface shear stress can be estimated as $\tau_{0}=\varrho_{a} C_{D} U_{10}^{2}$ dyn $\mathrm{cm}^{-2}$, where $\varrho_{a}$ is the density of air $\left(1.177 \times 10^{-3} \mathrm{~g} \mathrm{~cm}^{-3}\right), C_{D}$ (dimensionless) is the wind drag coefficient $\left(=1.3 \times 10^{-3}\right.$, but somewhat depen-

\begin{tabular}{|c|c|c|c|c|}
\hline Region & $\begin{array}{c}\text { Attenuation } \\
\text { coefficient } \\
\mathrm{k}\left(\mathrm{m}^{-1}\right)\end{array}$ & $\begin{array}{c}\text { Vertical } \\
\text { diffusivity } \\
\mathrm{K}_{2}\left(\mathrm{~cm}^{2} \mathrm{~s}^{-1}\right)\end{array}$ & $\begin{array}{c}\text { Incubation } \\
\text { time } \\
t(h)\end{array}$ & $t^{*}$ \\
\hline Oligotrophic ocean & 0.05 & $0.1-10$ & 12 & $10^{-3}-10^{-1}$ \\
\hline Temperate ocean & 0.15 & $1-100$ & 4 & $3 \times 10^{-2}-3 \times 10^{0}$ \\
\hline Coastal ocean & $0.31-1$ & $1-100$ & 4 & $4 \times 10^{-1}-10^{2}$ \\
\hline
\end{tabular}

Table 1. Estimated range (order of magnitude) of dimensionless parameter, $t^{*}$, in surface mixed layer of various regions 
dent on wind speed) and $\mathrm{U}_{10}\left(\mathrm{~cm} \mathrm{~s}^{-1}\right)$ is the wind speed measured at $10 \mathrm{~m}$ above the water surface. The mixing length scale is the lesser of the mixed layer depth or the Eckman layer thickness (Denman \& Gargett 1983). For tidal flow a similar formulation would hold for the diffusivity, but the shear velocity and length scale would be estimated from properties of the mean flow (Uncles \& Joint 1983). In a stratified layer the diffusivity is approximated by $\mathrm{K}_{z}=0.25 \varepsilon / \mathrm{N}^{2}$ (Denman \& Gargett 1983) where $\varepsilon$ is the rate of turbulent energy dissipation and $\mathrm{N}^{2}$ is the square of the Brunt-Vaisala frequency. In this form the length scale over which mixing takes place is contained implicitly in that the largest eddy that can exist before being suppressed by the buoyancy is also governed by the dissipation and $N^{2}$ (Denman \& Gargett 1983). Dissipation can be estimated from microstructure measurements (Dillon \& Caldwell 1980, Oakey 1982), or from windspeed (Oakey \& Elliott 1982), and $\mathrm{N}^{2}$ is obtained from routine temperature and salinity profiles.

Phytoplankton photosynthesis in fluctuating light. The second broad condition for obtaining discrepancies between measured rates of production in mixed versus statically-contained populations is that parameters of the P-I relation must be time dependent. Time dependence in itself is not a sufficient condition, for Gallegos et al. (1977) and Marra \& Heinemann (1982) observed fluctuations in rates of oxygen production that were proportional to the rate of change of irradiance and could be modeled by a linear differential equation. The photosynthetic response was symmetrical in increasing and decreasing light in these studies, so that the time integral of production could not be affected by that particular form of time dependence. If it actually occurs, enhancement of integral photosynthesis by vertical mixing must then depend on a nonlinear interaction between photosynthesis and fluctuating light.

Fluctuations in rates of oxygen production with nonlinear kinetics capable of enhancing integral photosynthesis have been measured by Harris \& Piccinin (1977) using concentrated field populations and by Marra (1978a, 1980) using laboratory cultures. In these experiments the rate of oxygen production in the first few minutes after exposure to saturating or inhibiting irradiance was much higher than the average rate measured over several hours. A quantitative index of the extent to which photosynthesis is biased in constant light might be the ratio of the maximum shortterm rate to the long-term averaged rate. However, because time series of oxygen evolution cannot be measured at naturally occurring cell densities, such a ratio could not be measured in routine field experiments.

In published experiments in which some form of vertical motion was supplied, enhancement of integrated photosynthesis in the mixed population relative to stationary populations has been observed only when the stationary population was susceptible to photoinhibition at surface intensities (Jewson \& Wood 1975 Marra 1978b, Gallegos \& Platt 1982). This is a qualitative correspondence only, for elimination of photoinhibition by itself was insufficient to account for the quantitative difference between the 2 integrals when differences were found (Marra 1978b, Gallegos \& Platt 1982). We quantify the susceptibility to photoinhibition at surface intensities using a modification of the equation of Platt et al. (1980),

$$
\mathrm{P}(\mathrm{I})=\mathrm{P}_{\mathrm{s}}\left[1-\exp \left(-\mathrm{I} / \mathrm{I}_{\mathrm{s}}\right)\right] \exp \left(-\mathrm{I} / \mathrm{I}_{\mathrm{b}}\right)
$$

where $P_{s}$ controls the vertical scaling and $I_{s}$ and $I_{b}$ control the onset of light saturation and photoinhibition respectively. The parameter $\mathrm{P}_{\mathrm{s}}$ has been interpreted as a potential light-saturated rate of photosynthesis that would be obtained if photoinhibition were absent, and is greater than the maximum observed rate, $\mathrm{P}_{\mathrm{m}}$, whenever $\mathrm{I}_{\mathrm{b}}<\infty$ (Platt \& Gallegos 1980, Platt et al. 1980). This implies that photoinhibition acts over all light intensities whenever Eq. (6) holds. Vincent et al. (1984) found experimental evidence for such an interpretation, and a similar explanation was given by Megard et al. (1984) for an analogous parameter in an equation for oxygenic photosynthesis.

We derive an index for the effects of vertical motion on depth-integrated photosynthesis by assuming that vertical mixing, by moving cells into and out of the near-surface region before the effects of photoinhibition become pronounced, allows the phytoplankton to produce at rates intermediate between $\mathrm{P}_{\mathrm{s}}$ and the rate

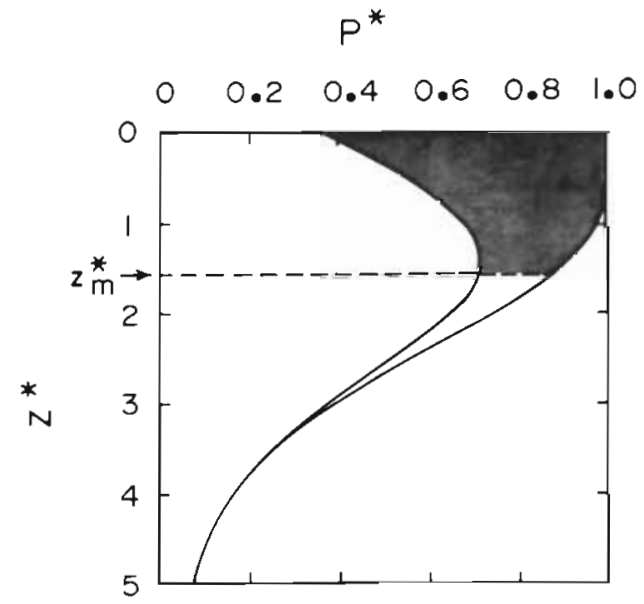

Fig. 1. Normalized profiles of photosynthesis as a function of optical depth for curves with $\left(I_{b}\right)^{-1}=1$, and without $\left(I_{b}{ }^{\prime}\right)^{-1}=0$ photoinhibition. $I_{s}{ }^{*}=0.2$ in both. Shaded region is the integral over the optical mixed depth $\left(z_{m}{ }^{*}\right)$ of the difference between 2 curves, and is an index of the potential for vertical mixing to enhance integral photosynthesis 
actually measured in statically-fixed incubations. (This differs from the analysis of Farmer \& Takahashi [1982] who used $\mathrm{P}_{\mathrm{m}}$ as an upper bound.) The maximum percent enhancement of depth-integrated photosynthesis can be calculated by determining the area, from the surface to the mixed-layer depth, between photosynthesis-depth profiles produced by Eq. (7) with $\left(\mathrm{I}_{\mathrm{b}}{ }^{-1}>0\right)$ and without $\left(\mathrm{I}_{\mathrm{b}}{ }^{-1}=0\right)$ photoinhibition (Fig. 1). To do this, Eq. (6) is non-dimensionalized by normalizing photosynthesis to $\mathrm{P}_{\mathrm{s}}$ and by normalizing irradiance $I$ and the irradiance parameters $I_{s}$ and $I_{b}$ to the surface incident irradiance, $\mathrm{I}_{0}$. The non-dimensionalized form is

$$
\mathrm{P} \cdot\left(\mathrm{I}^{\bullet}\right)=\left[1-\exp \left(-\mathrm{I}^{*} / \mathrm{I}_{\mathrm{s}}{ }^{*}\right)\right] \exp \left(-\mathrm{I}^{*} / \mathrm{I}_{\mathrm{b}}{ }^{*}\right)
$$

where $\mathrm{P}^{*}=\mathrm{P} / \mathrm{P}_{\mathrm{s}} ; \mathrm{I}^{*}=\mathrm{I} / \mathrm{I}_{0} ; \mathrm{I}_{\mathrm{s}}{ }^{*}=\mathrm{I}_{\mathrm{s}} / \mathrm{I}_{0} ; \mathrm{I}_{\mathrm{b}}{ }^{*}=\mathrm{I}_{\mathrm{b}} / \mathrm{I}_{0}$. In constructing depth profiles using Eq. (7), depth is nondimensionalized by scaling to the attenuation length, $z^{*}=k z$. The area of the shaded region in Fig. 1, which we denote $f\left(I_{s}{ }^{*}, I_{b}{ }^{*}, z_{m}{ }^{*}\right)$, is given by

$$
\begin{aligned}
\mathrm{f}\left(\mathrm{I}_{\mathrm{s}}^{*}, \mathrm{I}_{\mathrm{b}}{ }^{*}, \mathrm{z}_{\mathrm{m}}{ }^{*}\right)=\int_{0}^{\mathrm{z}_{\mathrm{m}}{ }^{*}}\left\{1-\exp \left[-\exp \left(-\mathrm{z}^{*}\right) / \mathrm{I}_{\mathrm{s}^{*}}\right]\right\} \\
\quad\left\{1-\exp \left[-\exp \left(-\mathrm{z}^{*}\right) / \mathrm{I}_{\mathrm{b}}{ }^{*}\right]\right\} \mathrm{dz} \cdot
\end{aligned}
$$

where $z_{m}{ }^{*}=k z_{m}$. Noting that $I^{*}=e^{-z^{*}}$, and that $\mathrm{dz}^{*}=\mathrm{dI}^{*} / \mathrm{I}^{*}$ and making the appropriate change in the limits of integration, the integral in Eq. (8) is given by

$$
\begin{aligned}
& f\left(I_{s} \cdot I_{b} \cdot, z_{m}{ }^{*}\right)=\sum_{n=1}^{\infty} \\
& \frac{(-1)^{n}\left(1-x_{m}{ }^{n}\right)\left\{\left[\left(I_{s}^{*}\right)^{-1}+\left(I_{b}{ }^{*}\right)^{-1}\right]^{n}-\left[\left(I_{s} \cdot\right)^{-n}+\left(I_{b} \cdot\right)^{-n}\right]\right\}}{n \cdot n !}
\end{aligned}
$$

where $\mathrm{x}_{\mathrm{m}}=\exp \left(-\mathrm{z}_{\mathrm{m}} \cdot\right)$. We regard this area as an index of the potential for vertical mixing to enhance depth-integrated photosynthesis. The degree to which this potential is realized depends on how effective the vertical mixing is at maintaining photosynthetic performance close to that defined by Eq. (7) with $\left(\mathrm{I}_{\mathrm{b}}{ }^{*}\right)^{-1}=\mathrm{O}_{\text {; }}$ it may therefore be a function of $\mathrm{t}^{*}$ or the temporal variability of $I_{0}$. (Note that any effects of variations in $I_{0}$ depend only on the method of determining photosynthetic rates. Normalizing the intensity parameters to $I_{0}$ does not eliminate any effects of variations in $I_{0}$ for in situ or simulated in situ incubations, as any such effects are already incorporated in measured photosynthetic rates.) An upper bound to the percent enhancement of depth-integrated photosynthesis is given by $100 \mathrm{f}\left(\mathrm{I}_{\mathrm{s}}{ }^{\circ}, \mathrm{I}_{\mathrm{b}}{ }^{\circ}, \mathrm{z}_{\mathrm{m}}{ }^{\circ}\right) / \mathrm{g}\left(\mathrm{I}_{\mathrm{s}}{ }^{\circ}, \mathrm{I}_{\mathrm{b}}{ }^{*}, \mathrm{z}_{\mathrm{m}}{ }^{\circ}\right)$, where $\mathrm{g}\left(\mathrm{I}_{\mathrm{s}}{ }^{*}, \mathrm{I}_{\mathrm{b}}{ }^{*}, \mathrm{z}_{\mathrm{m}}{ }^{*}\right)$ is the area to the left of the photosynthesis-depth profile with photoinhibition, and is given by

$$
\begin{aligned}
& \mathrm{g}\left(\mathrm{I}_{\mathrm{s}} \cdot, \mathrm{I}_{\mathrm{b}}{ }^{*}, \mathrm{z}_{\mathrm{m}} \cdot\right)= \\
& \quad \sum_{\mathrm{n}=1}^{\infty} \frac{(-1)^{\mathrm{n}}\left(1-\mathrm{x}_{\mathrm{m}}{ }^{n}\right)\left\{\left(\mathrm{I}_{\mathrm{b}} \cdot\right)^{-n}-\left[\left(\mathrm{I}_{\mathrm{s}} \cdot\right)^{-1}+\left(\mathrm{I}_{\mathrm{b}} \cdot\right)^{-1}\right]^{n}\right\}}{\mathrm{n} \cdot \mathrm{n} !}
\end{aligned}
$$

Comparison with published data. Data from which the specific variance of light during an incubation can be calculated are to found in studies by Jewson \& Wood (1975), Marra (1978b), Gallegos \& Platt (1982), and Yoder et al. (1984, pers. comm.). We use the advective term (i.e. $\mathrm{k}^{2} \operatorname{Var}[z]$ ) to represent the total variance of the light field during the incubations. This is valid because Marra (1978b) reported that his experiments were done under clear skies; Gallegos \& Platt (1982) used production estimates from an incubator as their control; as will be shown below, the variability in light imposed by the experimental design of Jewson \& Wood (1975) was so great that variations in cloud cover would be only a marginal addition to the total variance. Gallegos \& Platt (1982) reported simulated diffusivities for their experiments which simulated random transport in a light gradient. The variance of the light field for those experiments is given by $k^{2} K_{z} t$. For other experiments simulated velocities were reported, so that the variance of light is given by $\mathrm{k}^{2} \mathrm{wht}$. For the experiments of Gallegos \& Platt (1982) and of Jewson \& Wood (1975), the dimensionless irradiance parameters were estimated by nonlinear least squares parameter estimation (Gallegos \& Platt 1981) using data from the static control incubations. For the other studies, and for examination of regional variations, the number of points were too few for nonlinear parameter estimation. In these cases, the irradiance parameters were estimated by solution of Eq. (7) from measured values of percent inhibition at the surface and the optical depth of optimal photosynthesis.

\section{RESULTS AND DISCUSSION}

Values for the dimensionless irradiance parameters $I_{\mathrm{s}}{ }^{*}$ and $\mathrm{I}_{\mathrm{b}}{ }^{*}$ are presented in Table 2 , along with the depth integrals $f\left(I_{s}{ }^{*}, I_{b}{ }^{*}, z_{m}{ }^{*}\right)$ and $g\left(I_{s}{ }^{*}, I_{b}{ }^{*}, z_{m}{ }^{*}\right)$ for published experiments where simulated vertical mixing increased depth-integrated photosynthesis. Also shown are the computed maximum potential and the observed percent enhancement. In two of the experiments, one by Marra (1978b) and one by Gallegos \& Platt (1982), the observed percent enhancement exceeded the computed maximum potential. These discrepancies may be due to experimental procedures. Agitation of phytoplankton caused by simulated mixing may stimulate production in some algal assemblages independently of the effects of light fluctuations 
Table 2. Dimensionless irradiance parameters, depth integrals, and computed maximum percent enhancement estimated from published experiments in which simulated vertical movement enhanced integral photosynthesis

\begin{tabular}{|c|c|c|c|c|c|c|}
\hline \multirow[b]{2}{*}{ Identifier } & \multirow[b]{2}{*}{$I_{s}{ }^{\prime}$} & \multirow[b]{2}{*}{$I_{b}^{*}$} & \multirow[b]{2}{*}{$f\left(I_{s^{*}}, I_{b}{ }^{*}, z_{m}{ }^{*}\right)$} & \multirow[b]{2}{*}{$g\left(I_{s}{ }^{*}, I_{b}{ }^{*}, z_{m}{ }^{*}\right)$} & \multicolumn{2}{|c|}{ Percent enhancement } \\
\hline & & & & & Max. & Obs. \\
\hline 20 Apr $1972^{\ddagger}$ & 0.300 & 0.585 & 0.778 & 0.914 & 85.1 & 29.0 \\
\hline $5 \mathrm{Aug}^{\mathrm{b}}$ & 0.183 & 1.445 & 0.307 & 0.527 & 58.3 & 46.5 \\
\hline 19 Aug $^{b}$ & 0.402 & 0.607 & 0.608 & 0.464 & 131.0 & 30.8 \\
\hline $17 \mathrm{Sep}^{\mathrm{b}}$ & 0.490 & 0.414 & 0.729 & 0.414 & 176.1 & $18.6^{\mathrm{d}}$ \\
\hline 28 Oct $^{\mathrm{b}}$ & 0.310 & 1.908 & 0.315 & 1.318 & 23.9 & 87.5 \\
\hline Sta. $4^{c}$ & 0.100 & 3.200 & 0.259 & 2.552 & 10.1 & 30.1 \\
\hline Sta. $67^{c}$ & 0.067 & 0.632 & 0.901 & 1.009 & 89.3 & 76.3 \\
\hline Sta. $81^{c}$ & 0.063 & 1.609 & 0.483 & 2.032 & 23.8 & $9.4^{\mathrm{d}}$ \\
\hline Sta. $129^{c}$ & 0.050 & 1.450 & 0.540 & 2.236 & 24.2 & 24.4 \\
\hline \multirow{2}{*}{\multicolumn{7}{|c|}{ a Jewson \& Wood (1975) }} \\
\hline \multicolumn{3}{|c|}{ b Marra (1978b) } & & & & \\
\hline \multicolumn{7}{|c|}{ ' Gallegos \& Platt (1982) } \\
\hline d Significant a & nfidenc & 11 other & ificant at $95 \%$ le & & & \\
\hline
\end{tabular}

on photoinhibition, perhaps by disrupting diffusion gradients at the cell surface. Three of the 47 experiments reported by Yoder et al. (1984) resulted in enhanced photosynthetic rates although there was no observed photoinhibition in the static incubation; these too may have been subject to artifacts of manipulation. Errors in parameter estimates may also result in underestimation of the computed maximum percent enhancement, although it is doubtful that random errors in parameters could result in differences as large as those observed. Alternatively, the assumptions of the analysis may be flawed; perhaps the maximum rate of photosynthesis achieved in a fluctuating light field is higher than that estimated by $P_{s}$. If true, the magnitude of the integral $f\left(\mathrm{I}_{\mathrm{s}}{ }^{*}, \mathrm{I}_{\mathrm{b}}{ }^{*}, \mathrm{z}_{\mathrm{m}}{ }^{*}\right)$ still correctly predicts the qualitative occurrence of enhanced integral photosynthesis, even though the quantity is underestimated. Further analysis will be confined to such qualitative correspondence.
In Fig. 2 the results of experiments in which vertical mixing was simulated are plotted with respect to the 2 dimensionless parameters $t^{*}$ and $f\left(I_{s}{ }^{*}, I_{b}{ }^{*}, z_{m}{ }^{*}\right)$. Filled symbols are cases in which simulated mixing had a significant positive effect on integrated production estimates. These results suggest that there may be a critical region where the critical value of $f\left(\mathrm{I}_{\mathrm{s}}{ }^{\circ}, \mathrm{I}_{\mathrm{b}}{ }^{\circ}, \mathrm{z}_{\mathrm{mn}}{ }^{*}\right)$ has a minimum in the neighborhood of $t^{*} \simeq 10$. This represents a large degree of variability in the light field of the uncontained population and is likely to be encountered only in coastal regions, estuaries, and eutrophic lakes (Table 1). The minimum critical value of $f\left(I_{s}{ }^{*}, I_{b}{ }^{*}, z_{m}{ }^{*}\right)$ is about 0.2 . At the extremes of $t^{*}$ $\left(<10^{-1}\right.$ and $\left.>10^{2}\right)$ the critical value of $f\left(I_{s}{ }^{*}, I_{b}{ }^{*}, z_{m}{ }^{*}\right)$ is about 1 .

The integral $\mathrm{f}\left(\mathrm{I}_{\mathrm{s}}{ }^{*}, \mathrm{I}_{\mathrm{b}}{ }^{*}, \mathrm{z}_{\mathrm{m}}{ }^{*}\right)$ is difficult to compute, as it involves testing for convergence of an infinite series, and requires knowledge of the optical depth of the mixed layer, which is not always given in published

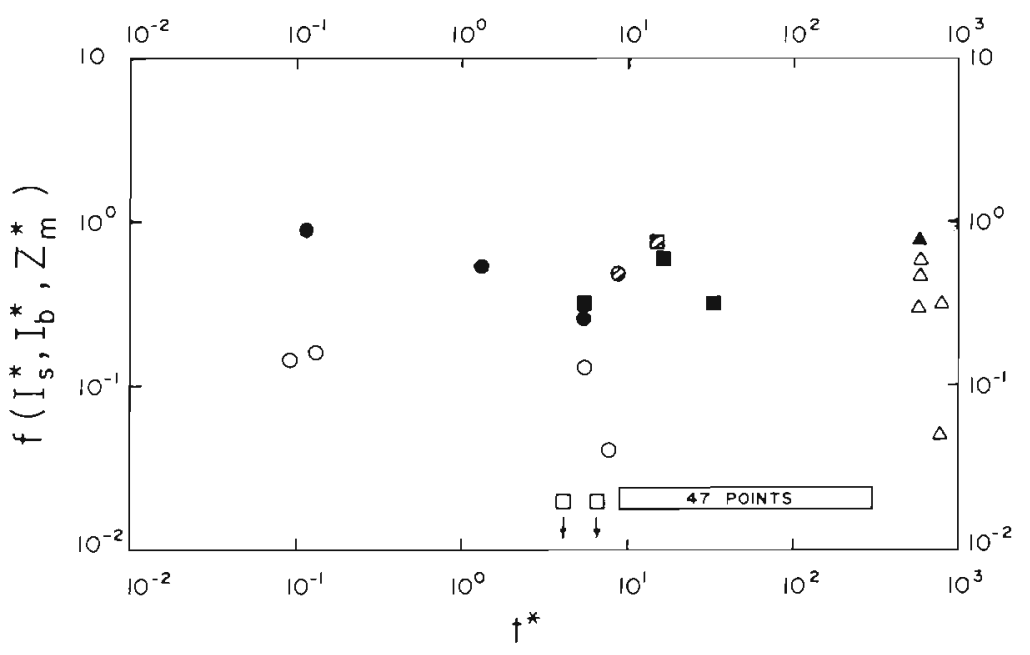

Fig. 2. Experiments in which vertical mixing was simulated, plotted with respect to the dimensionless parameters, $t^{*}$ and $\left(I_{b}{ }^{*}\right)^{-1}$. Triang. les correspond to data of Jewson \& Wood (1975); squares: Marra (1978b); circles: Gallegos \& Platt (1982); block of 47 points: Yoder et al. (1984). Closed or hatched symbols indicate experiments in which vertical mixing enhanced integral photosynthesis ( $P>0.95$ or $\mathrm{P}>0.90)$, open symbols indicate those in which simulated mixing had no effect or depressed integral photosynthesis 


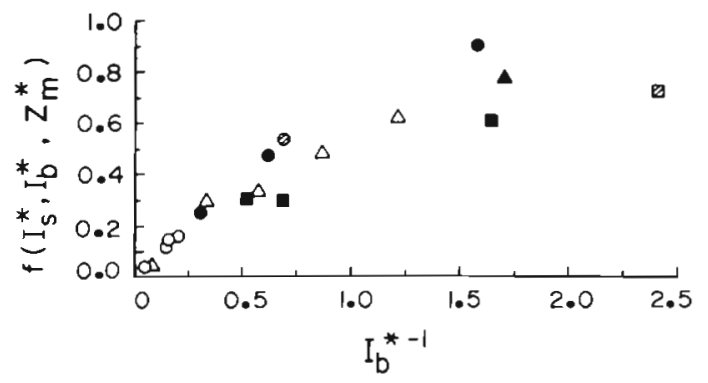

Fig. 3. $f\left(I_{s}{ }^{*}, I_{b}{ }^{*}, z_{m}{ }^{*}\right)$ (area of shaded region in Fig. 1) as a function of $\left(\mathrm{I}_{\mathrm{b}}{ }^{*}\right)^{-1}$. Symbols as in Fig. 2

data sets. We therefore seek an index with approximately the same information content as $\mathrm{f}\left(\mathrm{I}_{5}{ }^{*}, \mathrm{I}_{\mathrm{b}}{ }^{*}, \mathrm{z}_{\mathrm{m}}{ }^{*}\right)$ but which can be evaluated directly from $P$ vs I data. A plot of $f\left(I_{s}{ }^{*}, I_{b}{ }^{*}, z_{m}{ }^{*}\right)$ against the inverse irradiance parameter $\left(I_{b}\right)^{-1}$ (Fig. 3) shows that the area of the shaded region in Fig. 1 is governed strongly by the value of $\left(I_{b}^{*}\right)^{-1}$. In particular, the relation is very precise in the range of $f\left(I_{s}{ }^{*}, I_{b}{ }^{\circ}, z_{m}{ }^{\circ}\right)<0.3$, which contains the minimum critical value of $f\left(\mathrm{I}_{\mathrm{s}}{ }^{*}, \mathrm{I}_{\mathrm{b}}{ }^{*}, \mathrm{z}_{\mathrm{m}}{ }^{*}\right)$. This suggest that $\left(\mathrm{I}_{\mathrm{b}}{ }^{*}\right)^{-1}$ may be substituted for $\mathrm{f}\left(\mathrm{I}_{\mathrm{s}}{ }^{*}, \mathrm{I}_{\mathrm{b}}{ }^{*}, \mathrm{z}_{\mathrm{m}}{ }^{*}\right)$ without loss of generality.

In Fig. 4 the experiments in which vertical mixing was simulated are re-plotted as a function of $t^{*}$ with $\left(\mathrm{I}_{\mathrm{b}}{ }^{*}\right)^{-1}$ substituted for $\mathrm{f}\left(\mathrm{I}_{\mathrm{s}}{ }^{*}, \mathrm{I}_{\mathrm{b}}{ }^{*}, \mathrm{z}_{\mathrm{m}}{ }^{*}\right)$. Again, a critical region can be identified which closely resembles that found in Fig. 2. The minimum critical value of $\left(\mathrm{I}_{\mathrm{b}}{ }^{*}\right)^{-1}$ is about 0.25 , and occurs at $t^{*} \simeq 10$. At the extremes of $t^{*}$ the critical value of $\left(I_{b}{ }^{-1}\right)^{-1}$ is about 1.5 .

Two cautions are in order. First, in none of the experiments was there direct physical evidence that the simulated intensities of mixing were representative of in situ conditions at the time of sampling; second, there are large regions of the plane that are poorly sampled. In particular, there is a marked lack of samples showing susceptibility to photoinhibition among experiments in which $t^{*}$ varied from $10^{1}$ to $10^{3}$ (i.e. typical of coastal conditions). Thus, the boundaries of the critical region remain poorly defined. Nevertheless, a minimum with respect to $t$ " might be defended for physiological reasons. At the highest values of $t^{*}$ such as simulated by Jewson \& Wood (1975), the algal cells are advected through the light gradient so rapidly that the highest rates of photosynthesis may not be achieved due to time lags in the photosynthetic response (Harris \& Piccinin 1977). This would especially be true when mixing carried the organisms well below the euphotic zone. At the lowest values of $t^{*}$ the phytoplankton experience much less variability in light, so that a much greater time dependence (modeled here as susceptibility to photoinhibition) is required to produce a measurable effect. Thus, a minimum critical value of $\left(\mathrm{I}_{\mathrm{b}}{ }^{*}\right)^{-1}$ can be expected at some intermediate value of $t^{*}$

Although the exact shape of the boundaries of the critical value of $\left(\mathrm{I}_{\mathrm{b}}\right)^{-1}$ as a function of $\mathrm{t}^{*}$ may be altered by future observations, the following definitive regions may be identified from existing data, and these may prove to be robust. For $\left(\mathrm{I}_{\mathrm{b}}{ }^{\bullet}\right)^{-1}<0.25$, vertical advection has no effect on productivity estimates due to a lack of susceptibility to photoinhibition. For $\left(\mathrm{I}_{\mathrm{b}}{ }^{*}\right)^{-1}$ $>1.5$ any vertical motion at all is sufficient to enhance productivity over the static-bottle estimate, due to the extreme susceptibility to surface inhibition. For $0.25 \leq$ $\left(\mathrm{I}_{\mathrm{b}}{ }^{*}\right)^{-1} \leq 1.5$, the effect of vertical advection on productivity estimates requires explicit knowledge of the physical conditions existing during the incubation.

For comparison with these boundaries, the distribution of $\left(I_{b}\right)^{-1}$ in data sets from 4 regions is illustrated in Fig. 5. In a seasonal study of St. Margaret's Bay, a small coastal embayment (Platt \& Irwin 1968), $80 \%$ of the in situ incubations had surface photosynthesis maxima (Fig. 5 A), suggesting little effect of containment at fixed depths. On the continental shelf off Nova
Fig. 4. As Fig. 2, with $\left(\mathrm{I}_{b}\right)^{-1}$ substituted for $f\left(I_{s} \cdot, I_{b}{ }^{*}, z_{m} \cdot\right)$

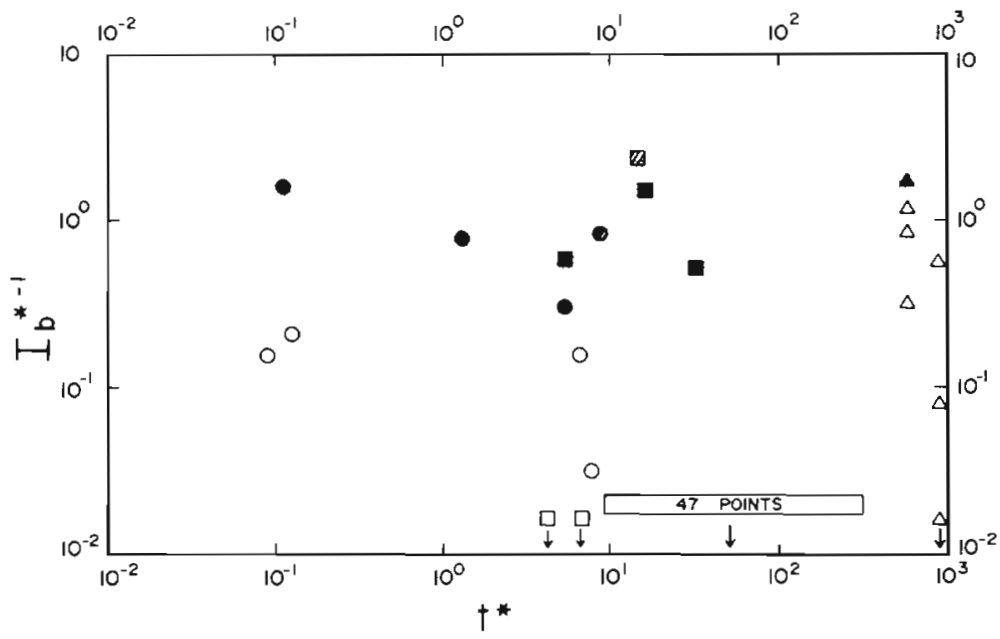



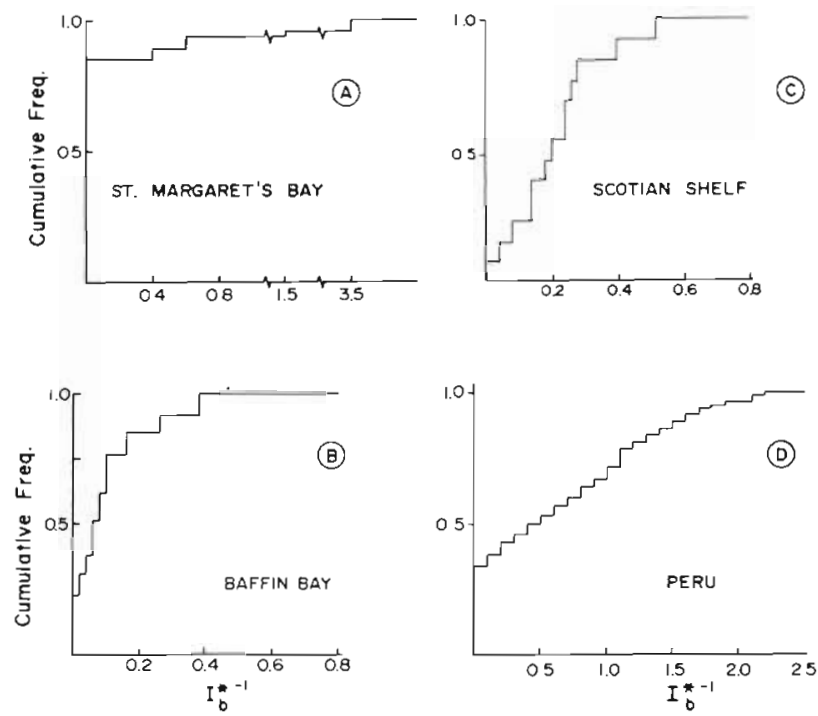

Fig. 5. Cumulative distributions of $\left(I_{b}{ }^{*}\right)^{-1}$ estimated from data sets from 4 regions: (A) St. Margaret's Bay, Nova Scotia; (B) continental shelf off Nova Scotia; (C) Baffin Bay, eastern Canadian arctic; (D) Peruvian upwelling region

Scotia during the spring of 1979 and after establishment of the seasonal pycnocline, $85 \%$ of the samples from the upper layer had values for $\left(\mathrm{I}_{b}{ }^{*}\right)^{-1}$ less than the 0.25 minimum critical value; the remaining $15 \%$ were in the range that requires knowledge of $t^{*}$ in order to judge whether there were effects due to static containment. In a set of experiments from Baffin Bay (Fig. 5 C), a region with a strong seasonal pycnocline (Platt et al. 1982), $85 \%$ of samples from the mixed layer had values of $\left(I_{b}\right)^{-1}$ less than 0.25 ; the remaining values were less than 0.4, again suggesting little effect of vertical movement (see also Gallegos \& Platt 1982). The greatest range of values for $\left(\mathrm{I}_{b}{ }^{*}\right)^{-1}$ in the data sets examined was from the Peru upwelling region (Fig. 5 D; Barber et al. 1978). In that region, $38 \%$ of the incubations had surface maxima; the remaining values are distributed fairly uniformly between 0.1 and 2.0 , with about $50 \%$ falling within the region requiring knowledge of $t^{*}$, and only about $12 \%$ above the 1.5 limit that is nearly certain to initiate bias.

It is of interest that the upwelling region off Peru would have the greatest range of variability in $\left(I_{b}{ }^{*}\right)^{-1}$. The data were taken on separate cruises over several years, and include numerous onshore-offshore transects. The data set therefore represents a wide range of physical conditions and biological communities. The correspondence in variability between the physical regime and $\left(I_{b}{ }^{*}\right)^{-1}$ underscores the importance of knowing the mixing characteristics prevailing during an experiment before drawing any conclusions about the artifactual nature of surface inhibition. If the criteria developed here prove to be robust, routine observations of weather conditions, water transparency, and P-I parameters can be used to estimate whether bias has been incurred by incubating in static bottles. More importantly, if bias is suspected, incubation procedures that incorporate light fluctuations (e.g. Gallegos \& Platt 1982) can be employed and the relations of Denman \& Gargett (1983) used to make the appropriate scaling of the simulated mixing.

The best predictor of enhancement due to mixing was the parameter $\left(I_{b}{ }^{*}\right)^{-1}$. The dependence on $t^{*}$ is relatively weak, and as discussed, the boundaries illdefined. Thus, the factors that determine whether or not mixing enhances photosynthesis are principally those that determine whether a sample taken from the mixed layer will be susceptible to photoinhibition. Although we have attempted to restrict the analysis to events occurring on the time scale of an incubation (during which parameters of the P-I response are presumed stationary), the longer term history of a population's exposure to light and the consequent adaptive changes in the P-I response must also be considered.

It is not uncommon for populations isolated below a seasonal thermocline to be susceptible to photoinhibition when exposed to surface conditions, while those taken within the mixed layer may show no susceptibility to photoinhibition (see Platt et al. 1982, Gallegos et al. 1983). Entrainment into the mixed layer of cells previously growing below a pycnocline may be one mechanism by which samples from the mixed layer may show susceptibility to photoinhibition, and this may have occurred in the experiments listed in Table 1. Only one of the points in the data of Gallegos \& Platt (1982) labeled as significantly enhanced by mixing was actually sampled from within the mixed layer (i.e. Table 1, Sta. 4); the others were taken from below a strong pycnocline. Furthermore, the sample from Sta. 4 was taken during a storm which deepened the mixed-layer, so that the sample may have contained populations susceptible to photoinhibition by virtue of their previous growth at low irradiance.

Susceptibility to photoinhibition might also develop in situ under conditions of relatively weak mixing, if the time scale of parameter adjustment (adaptation) is fast compared to the time scale of mixing. Lewis et al. (1984a) showed that the formation of gradients of photoadaptive properties in a mixed layer depends on 2 dimensionless groups, the optical depth of the mixed layer, $\mathrm{kh}$, and a ratio of adaptation time: mixing time, $\mathrm{K}_{\mathrm{z}} / \gamma \mathrm{h}^{2}$, where $\gamma$ is an adaptation time constant. Lewis (1984) plotted percent enhancement from Marra's data against the rate of dissipation of turbulent kinetic energy, $\varepsilon$, estimated from wind shear records, and found that enhancement decreased with increasing $\varepsilon$, and that the scaling was similar to that for formation of gradients in $\mathrm{P}_{\mathrm{m}}$ (Lewis et al. 1984b). That is, the great- 
est enhancement was found on the days with the least wind forcing.

\section{CONCLUSIONS}

That the results of simulated mixing experiments should be so equivocal is not surprising. From the analysis presented here and in Lewis et al. (1984a), we identify 3 categories of mixing and their probable effects on productivity estimates. Under conditions of moderate forcing which is strong enough to keep the mixed layer well-stirred with respect to phytoplankton photosynthetic parameters, particularly $\mathrm{I}_{\mathrm{b}}$, simulated mixing should have little effect on productivity estimates because the population will be uniformly adapted to near-surface conditions (i.e. low $\left[\mathrm{I}_{b}{ }^{*}\right]^{-1}$ ). This was the normal situation in the data of Gallegos \& Platt (1982, mixed-layer samples), and Yoder et al. (1984). Static incubations performed during forcing strong enough to deepen the mixed-layer may underestimate photosynthesis if organisms previously grown at low irradiance levels are entrained into the mixed layer. Finally, under conditions of weak mixing, gradients in photosynthetic parameters, especially $\mathrm{I}_{\mathrm{b}}$, may form in an otherwise isopycnic layer. In this case simulated mixing may lead to enhanced estimates of photosynthesis compared to static-bottle estimates, but the results must be interpreted cautiously: the simulated mixing may be more intense than in situ conditions, and a sample from a single depth may not contain the full range of adaptations present in the mixed layer.

We have treated the rather narrow problem of estimating the effects of light fluctuations on photosynthesis in a well-mixed layer. Over much of the world's oceans and much of the growing season, the euphotic zone is deeper than the mixed layer, and subsurface chlorophyll maxima and layering of species may occur (Cullen \& Eppley 1981, Kiefer \& Kremer 1981, Venrick 1982). Internal wave activity in a stratified water column may effect large and rapid changes in perceived light intensity at the depth of the chlorophyll maximum (Haury et al. 1983). A model of the effects of light fluctuations in a stratified water column would have to treat, in addition to the effects considered here, vertical variations in photosynthetic parameters (Gallegos et al. 1983), vertical variations in diffuse attenuation coefficient (Lewis et al. 1983), and phytoplankton behavioral responses (Kamykowski 1979, Cullen et al. 1983).

Acknowledgements. We thank Dr. J. Yoder for kindly furnishing unpublished results and Dr. J. Marra for access to raw data. Drs. M. R. Lewis and A. Stewart gave valuable comments on the manuscript. Part of this work was conducted while CLG was supported by the Natural Sciences and Engineering Research Council (Canada).

\section{LITERATURE CITED}

Barber, R. T., Huntsman, S. A., Kogelschatz, J. E., Smith, W. O., Jones, B. H., Paul, J. C. (1978). Carbon, chlorophyll and light extinction from JOINT II 1976 and 1977. Data Report 49, Coastal Upwelling Ecosystems Analysis, IDOE

Cullen, J. J., Eppley, R. W. (1981). Chlorophyll maximum layers of the Southern California Bight and possible mechanisms of their formation and maintenance, Oceanologica Acta 4: 23-32

Cullen, J. J., Stewart, E., Renger, E., Eppley, R. W., Winant, C. D. (1983). Vertical motion of the thermocline, nitracline and chlorophyll maximum layers in relation to currents on the Southern California Shelf. J. mar. Res. 41. 239-262

Denman, K. L., Gargett, A. E. (1983). Time and space scales of vertical mixing and advection of phytoplankton in the upper ocean. Limnol. Oceanogr. 28: 801-815

Dillon, T. M., Caldwell, D. R. (1980). The Batchelor spectrum and dissipation in the upper ocean. J. geophys. Res, 85: 1910-1916

Farmer, D. M., Takahashi, M. (1982). Effects of vertical mixing on photosynthetic responses. Jap. J. Limnol. 43: 173-181

Frechette, M., Legendre, L. (1978). Photosynthese phytoplanctonique: reponse à un stimulus simple, imitant les variations rapides de la lumière engendrées par les vagues. J. exp. mar. Biol. Ecol. 32: 15-25

Gallegos, C. L., Hornberger, G. M., Kelly, M. G. (1977). A model of river benthic algal photosynthesis in response to rapid changes in light. Limnol. Oceanogr. 22: 226-233

Gallegos, C. L., Platt, T. (1981). Photosynthesis measurements on natural populations of phytoplankton: numerical analysis. Can. Bull. Fish. Aquat. Sci. 210: 103-112

Gallegos, C. L., Platt, T. (1982). Phytoplankton production and water motion in surface mixed layers. Deep Sea Res. 29: $65-76$

Gallegos, C. L., Platt, T., Harrison, W. G., Irwin, B. (1983). Photosynthetic parameters of arctic marine phytoplankton: vertical variations and time scales of adaptation. Limnol. Oceanogr. 28: 698-708

Harris, G. P. (1978). Photosynthesis, productivity and growth: the physiological ecology of phytoplankton. Arch. Hydrobiol. Beih. Ergeb. Limnol. 10: 1-171

Harris, G. P. (1980). The measurement of photosynthesis in natural populations of phytoplankton. In: Morris, I. (ed.) The physiological ecology of phytoplankton. Blackwell, Oxford, p. 129-187

Harris, G. P., Piccinin, B. B. (1977). Photosynthesis by natural phytoplankton populations. Arch. Hydrobiol. 80: 405-457

Haury, L. R., Wiebe, P. H., Orr, M. H., Briscoe, M. G. (1983). Tidally generated high-frequency internal wave packets and their effects on plankton in Massachusetts Bay. J. mar. Res. 41: 65-112

Holmes, R. W. (1957). Solar radiation. In: Treatise on marine ecology and paleoecology, vol. 1, Ecology. Mem. no. 67, geol. Soc. Am., p. 109-128

Jewson, D. H., Wood, R. B. (1975). Some effects on integral photosynthesis of artificial circulation of phytoplankton through light gradients. Verh. int. Verein. theor. angew. Limnol. 19: 1037-1044

Kamykowski, D. (1979). The growth response of a model Gymnodinium splendens in stationary and wavy water columns. Mar. Biol. 50: 289-303

Kiefer, D. A., Kremer, J. N. (1981). Origins of vertical patterns of phytoplankton and nutrients in the temperate, open ocean: a stratigraphic hypothesis. Deep Sea Res. 28: $1087-1105$ 
Lewis, M. R. (1984). Photoadaptation by algae in the upper mixed layer of the ocean. Ph.D. thesis, Dalhousie Univ.

Lewis, M. R., Cullen, J. J., Platt, T. (1983). Phytoplankton and thermal structure in the upper ocean: consequences of nonuniformity in the chlorophyll profile. J. geophys. Res. 88: $2565-2570$

Lewis, M. R., Cullen, J. J., Platt, T. (1984a). Relationships between vertical mixing and photoadaptation of phytoplankton; similarity criteria. Mar. Ecol. Prog. Ser. 15: 141-149

Lewis, M. R., Horn, E. P. W., Cullen, J. J., Oakey, N. S., Platt, T. (1984b). Turbulent motions may control phytoplankton photosynthesis in the upper ocean. Nature, Lond. 311: $49-50$

Marra, J. (1978a). Effect of short-term variations in light intensity on photosynthesis of a marine phytoplankton. A laboratory simulation study. Mar. Biol. 46: 191-202

Marra, J. (1978b). Phytoplankton photosynthetic response to vertical movement in a mixed layer. Mar. Biol. 46: 203-208

Marra, J. (1980). Vertical mixing and primary production. Brookhaven Symp. Biol. 31: 121-137

Marra, J., Heinemann, K. (1982). Photosynthesis response by phytoplankton to sunlight variability. Limnol. Oceanogr. 27: 1141-1153

Megard, R. O., Tonkyn, D. W., Senft, W. H. II. (1984). Kinetics of oxygenic photosynthesis in planktonic algae. J. Plankton Res. 6: 325-337

Oakey, N. S. (1982). Determination of the rate of dissipation of turbulent energy from simultaneous temperature and velocity shear microstructure measurements. J. phys. Oceanogr. 12: 171-185

Oakey, N. S., Elliott, J. A. (1977). Vertical temperature gra- dient structure across the Gulf Stream. J. geophys. Res. 82: $1369-1380$

Oakey, N. S., Elliott, J. A. (1982). Dissipation within the surface mixed layer. J. phys. Oceanogr. 12: 171-185

Platt, T., Gallegos, C. L. (1980). Modeling primary production. Brookhaven Symp. Biol. 37: 339-362

Platt, T., Gallegos, C. L., Harrison, W. G. (1980). Photoinhibition of photosynthesis in natural assemblages of marine phytoplankton. J. mar. Res. 38: 687-701

Platt, T., Harrison, W. G., Irwin, B., Horn, E. P., Gallegos, C. L. (1982). Photosynthesis and photoadaptation of marine phytoplankton in the Arctic. Deep Sea Res. 29: 1159-1170

Platt, T., Irwin, B. (1968). Primary productivity measurements in St. Margaret's Bay, 1967. Fish. Res. Bd Can. Tech. Rep. 77: $1-123$

Platt, T., Jassby, A. D. (1976). The relationship between photosynthesis and light for natural assemblages of coastal marine phytoplankton. J. Phycol. 12: 421-430

Simpson, J. J., Dickey, T. D. (1981). The relationship between downward irradiance and upper ocean structure. J. phys. Oceanogr. 11: 309-323

Uncles, R. J., Joint, I. R. (1983). Vertical mixing and its effects on phytoplankton growth in a turbid estuary. Can. J. Fish. Aquat. Sci. 40 (1 Suppl.): 221-228

Venrick, E. L. (1982). Phytoplankton in an oligotrophic ocean: observations and questions. Ecol. Monogr. 52: 129-154

Vincent, W. F., Neale, P. J., Richerson, P. J. (1984). Photoinhibition: algal responses to bright light during diel stratification and mixing in a tropical alpine lake. J. Phycol. 20: 201-211

Yoder, J, Bishop, S. S., Emmanuele, K. A. (1984). Effect of irradiance fluctuations on photosynthesis of coastal phytoplankton. Trans. Am. geophys. Un. (EOS) 64: 1092

This paper was submitted to the editor; it was accepted for printing on July 9, 1985 